\title{
CIUDADANÍA ESPECIAL: UN ANÁLISIS DE LA CARTA DE DERECHOS DE LAS PERSONAS CON IMPEDIMENTOS ${ }^{1}$
}

\author{
Carmen Milagros Vélez ${ }^{2}$
}

...by lending a psychoanalytical ear to the incommensurable singularity of the exclusion unlike others that handicapped people suffer from, it becomes obvious that it concerns us. Not necessarily because "it could happen to anyone", but because it is already inside me: in our dreams, our anxieties, our amorous and existential crisis, in the lack in being that overwhelms us when our resistances fall apart and the "interior castle" itself begins to crumble. Since to recognize it in myself will help me to discover the incommensurable subject within the deficient body, in order to build together the project of a shared life.

(Julia Kristeva, 2005: 15).

\section{Resumen}

El presente trabajo es un análisis discursivo, que ha consistido en intentar develar la construcción de ciudadanía que el Estado adscribe a las personas nombradas con impedimentos, y cómo, mediante sus estatutos legales y políticas públicas se promueve la manifestación de la diversidad al interior de este sector de la población que se le nombra personas con impedimentos o discapacidades. En particular se analiza la producción del sujeto femenino, nombrado con impedimentos, cómo una diversidad posible y sus posibilidades de ejercicio de la ciudadanía. Se presenta un análisis discursivo crítico, para intentar develar las relaciones de poder que expresa y sostiene esta pieza de ley. La intención ha sido explorar en que formas, la Carta de Derechos de las Personas con Impedimentos puede representar en su constitución un acto de exclusión de las personas con impedimentos de la

\footnotetext{
${ }^{1}$ Artículo basado en la investigación de disertación para el Programa Doctoral de la Escuela Graduada de Trabajo Social Beatriz Lassalle, de la Universidad de Puerto Rico en Río Piedras.

${ }^{2}$ Profesora Escuela de Salud Pública, Universidad de Puerto Rico, Recinto de Ciencias Médicas.
} 
categoría ciudadano, para ubicarle en una categoría de ciudadanía especial y sus posibles implicaciones. Además, se explora posibles producciones al interior del discurso, su relación con otros textos y discursos en torno a las personas con impedimentos y un intento de entender las formas en que los discursos legales y que luego se convierten en política pública construyen posibilidades de acceso al ejercicio de la ciudadanía, en poblaciones que han sido llamadas vulnerables y que han sido vulnerabilizadas mediante el uso de palabras que constituyen narrativas colectivas.

Descriptores: personas con discapacidades, derechos civiles, análisis de discurso.

\section{Abstract}

The present article is a discourse analysis, that intends to reveal the social constructions of citizenship the State assigns people named with disabilities, and how diversity within the population is acknowledged by means of Law and Public Policy. The social construction of women named with disability, as a possibility of diversity, is particularly analyzed in terms of the opportunities for citizenship it deems possible. A Critical Discourse Analysis is presented with the intention of revealing power relations expressed by the Declaration of Rights for Persons with Disabilities in Puerto Rico. The methodology was used as an attempt to explore how this legislation, in it's intention of providing recognition of people named with disabilities as citizens, excludes this part of the population from general citizenship, referring to a special and separate category of citizenship, and what this implies. Furthermore, explores the possible production within contemporary discourse, and the texts of the legislation, of people named with disabilities as citizens. It also addresses the means by which law and latter public policy that derives from it, promotes or is an obstacle to exercising civil rights for people that have, by being named with disabilities, become socially vulnerable. This vulnerability is produced through words that constitute general social narratives.

Key words: persons with disabilities, civil rights, discourse analysis. 


\section{Introducción}

El proyecto de una vida compartida, según expresa Kristeva (2005), no puede iniciarse sin un reconocimiento de las diferencias, esas, que nos habitan a todas y todos. Este reconocimiento debe darse a nivel macro por parte de las instituciones que legislan y desarrollan política pública para atender los asuntos de las diferencias del cuerpo. No basta con identificar al otro u otra, sino entender que somos este otro/a en algún momento de nuestras vidas como ciudadanos. El presente es el resultado de un ejercicio que ha consistido en intentar develar la construcción de ciudadanía que el Estado adscribe a las personas con impedimentos, y cómo, mediante sus estatutos legales y políticas públicas se promueve la manifestación de la diversidad al interior de este sector de la población que se le nombra personas con impedimentos o discapacidades ${ }^{3}$. En particular se analiza la producción del sujeto femenino, nombrado con impedimentos, como una diversidad posible y sus posibilidades de ejercicio de la ciudadanía bajo los derechos que consigna la Carta de Derechos de las Personas con Impedimentos en Puerto Rico (Ley de Puerto Rico \#238, 2004).

¿Por qué analizar discursivamente la Carta de Derechos de la Personas con Impedimentos (en adelante CDPI)? Quizás, la pregunta más oportuna sería; ¿Por qué legislar una Carta de Derechos de los y las personas que son nombradas con impedimentos o discapacidades en Puerto Rico? Ésta legislación de reciente aprobación representa la respuesta y posición del Estado en cuanto a las personas con impedimentos y cómo se concibe su ciudadanía. Sin embargo, en el campo del desarrollo de los derechos ciudadanos en Puerto Rico tiene vigencia una Carta de Derechos en la Constitución del Estado Libre Asociado, desde el 1952. Además, nos alberga una Declaración Universal de los Derechos Humanos de las Naciones Unidas, redactada en el 1948 que consigna derechos ciudadanos a "todos los miembros de la familia humana", según lee el texto de esta

${ }^{3}$ Persona con impedimentos y persona con discapacidades son los referentes de uso común en el diálogo contemporáneo al hablar de personas que han sido diagnosticadas, certificadas y se reconocen como diferentes del cuerpo y que ven su acceso al ejercicio de la ciudadanía impedido por las formas en que la sociedad los/as construyen. En Puerto Rico el consenso es en el uso del término personas con impedimentos, mientras que en América Latina, España y el Caribe lo más frecuente es encontrar personas con discapacidades. En el presente ensayo se hará referencia a ambas formas indistintamente. 
Declaración (Organización de Naciones Unidas, 1948). Provocada por las interrogantes anteriormente planteadas, en este trabajo me he dirigido a analizar los textos de la CDPI, para mirar el tipo de ciudadanía que produce para las personas que se nombran con impedimentos o discapacidades y como compara con otros documentos de derechos humanos.

Si entendemos ciudadanía como propone Hannah Arendt, "el derecho a tener derechos, que incluye el derecho a la diferencia" (Erroles y Ferreres, 2002: 22), sería importante ver qué perspectiva de lo que significa ser mujer nombrada con impedimentos produce la CDPI, como una entre las múltiples diversidades posibles. Una de las preguntas que me han guiado en este análisis es ¿cuál es la construcción de la persona nombrada con impedimentos, en particular las mujeres con impedimentos, que privilegia esta pieza de ley y cómo puede incidir en las posibilidades de este sector de la población en cuanto a su ejercicio de los derechos y deberes ciudadanos?

He propuesto, un análisis discursivo crítico para intentar develar las relaciones de poder que expresa y sostiene esta pieza de ley (Powers, 2001). La intención ha sido explorar en qué formas, la CDPI puede representar en su constitución un acto de exclusión de las personas con impedimentos de la categoría ciudadano, para ubicarle en una categoría de 'ciudadanía especial' y sus posibles implicaciones. Este intento por supuesto, tiene la intención de iniciar una pesquisa mas abarcadora, al interior de lo que significa ser una persona nombrada con impedimentos, en el plano del ejercicio de la ciudadanía. Plantea, incluso, la interrogante; ¿existe tal cosa como una ciudadanía diferenciada, o sólo se puede ser ciudadana o ciudadano en su plenitud de expresión universal?

\section{Carta de Derechos de las Personas con Impedimentos de Puerto Rico: Expresión de política pública con relación a las personas nombradas con impedimentos}

La CDPI de Puerto Rico en sus textos se pronuncia como la expresión máxima de política pública del Estado en relación a las personas, que son nombradas con impedimentos. Es por ello que se analizarán las construcciones de las personas nombradas con impedimentos que esta ley sostiene. ¿Cómo promueve esta un entendido de las Personas nombradas con impedimentos y ¿cómo éstas construcciones pueden incidir en las políticas públicas que surgen como resultados de la implantación de ésta? 
Genealogía de la Carta de Derechos de las Personas con Impedimentos (CDPI)

En Puerto Rico se han elaborado y aprobado un número considerable de leyes cuya intención menciona el 'bienestar de los impedidos'. En Leyes más recientes se observa un cambio, de referirse a impedidos, a la categoría personas con impedimentos. ¿A qué obedece este cambio, y en qué momento específicamente se da? Este sería tema de otro ensayo, sin embargo, para efectos de este trabajo es importante mencionar el asunto de cómo se nombran las personas que intentan ser beneficiadas por la leyes de protección a los ciudadanos con discapacidades en Puerto Rico en contraste con la forma de nombramiento de estas personas en la CDPI.

La evolución del discurso permite comprender y dar sentido a los cambios materiales. La palabra es acción, en tanto las acciones tienen que ser apalabradas/codificadas para existir, las palabras hacen la acción perceptible y le dan sentido. La palabra constituye la acción o materialidad. Entonces la palabra es la más importante herramienta de cambio social (Fairclough, 1993). En la palabra intencionada, ensayada, y "performed", una y otra vez. Y bien como plantea Althusser, "interpelando sentidos", se da la multiplicación de las opciones en las mismas palabras. Las palabras son acciones que a su vez producen otras acciones, en cadena, que van formando el entorno social de los seres humanos que afectan nuestras experiencias en sociedad. Las palabras, los objetos, que producen los seres humanos, forman textos que a su vez componen discursos que constituyen el entorno social y personal (Foucault, 1963).

La noción de impedimento o discapacidad en los seres humanos es constituida material y discursivamente (Corker y French, 2002). No hay lugar a duda que es abrumadoramente prevalente la construcción negativa de lo que tiene que ver con las diferencias humanas nombradas impedimentos o discapacidades, en el mundo occidental. Tampoco hay que dudar de la existencia de un cambio sustancial en las construcciones sociales que se elaboran sobre personas nombradas con impedimentos o discapacidades, que han facilitado para muchos un reconocimiento más abarcador de estas personas como ciudadanos y ciudadanas con derechos. Sin embargo, estos cambios han sido muy lentos e inconsistentes, tomando en consideración, que la lucha social y política por la igualdad de derechos de las personas con impedimentos, de forma intensa, data de mediados del siglo pasado (Braddock, citado en Albrecht, et al., 2001). Un ejemplo en Puerto Rico, de 
lo lento de este proceso de lucha es la demanda de clase Rosa Lydia Vélez vs. El Departamento de Educación de Puerto Rico, encausada hace casi 30 años y cuyas estipulaciones impone el Tribunal Superior de Puerto Rico en el 2002 , en el presente 2008, no son una realidad para los miles de niños y niñas nombrados y nombradas con discapacidades, que permanecen sin la atención apropiada para su desarrollo óptimo académico y social. Este estancamiento permite que se continúe manteniendo a niños/as y jóvenes, nombrados y nombradas con discapacidades en Puerto Rico, al margen de la ciudadanía. En un estudio reciente de la Comisión de Derechos Civiles de Puerto Rico se evidencian que no se cumplen los mandatos de esta acción de clase (Comisión de Derechos Civiles, 2006). La experiencia con esta acción de clase nos muestra además, que lo que ocurre en los tribunales y la legislatura por si sólo no cambia las realidades sociales. Llama la atención, que las reformas legales tienen que ir a la par con una reforma social y cultural en la que las construcciones desvaloradas sean sustituidas por unas más justas. Para ello es fundamental, revisar los discursos de las políticas públicas y de las fuentes mediáticas.

Algunos autores afirman que el cambio a nivel del discurso ha sido dominado por lo clínico y legitimado por lo legal, y que éste se centra en los defectos del cuerpo (físico y psíquico), en los diagnósticos (Mitchell \& Snyder, 1997). El modelo médico/clínico produce un discurso que objetiviza al sujeto nombrado con impedimentos, definiéndole como lo que le falta o lo que no es. La constitución del sujeto nombrado con impedimentos se da en función de lo que no es, interpretado como ser menos de lo que es esperado. Esta reducción impide el desarrollo de la persona que es nombrada con impedimentos o discapacidades como ciudadano/a, ya que le separa del entorno social general y sus posibilidades de acceso al ejercicio de la ciudadanía. El costo de las oportunidades de ejercer la ciudadanía es, disminuir y eliminar la diferencia para poder ser incluido/a, esencialmente invisibilizar su diferencia (Kristeva, 2005). Este es un discurso hegemónico que responde a intereses particulares que promueven su estatus, mediante la legitimación de una forma limitada de ser elegible o en este caso apto o apta para ejercer la ciudadanía. Las construcciones sociales vigentes en torno a lo que se considera un ciudadano no son estáticas, sin embargo responden a los intereses de las personas e instituciones que al presente se encuentran en el poder. En una sociedad de mercado, globalizada y mediatizada, este ciudadano hegemónico se presenta con unas características que incluyen no 
sólo las habilidades del cuerpo, sino una apariencia, que excluye aquellas que no funcionan dentro de los parámetros de lo esperado en su entorno social. El discurso médico clínico ha sido esgrimido como fundamento en el desarrollo de legislación y política pública que perpetua la visión de la persona nombrada con impedimentos como objeto clasificable por diagnóstico. No es difícil entender la procedencia de este discurso de dependencia e insuficiencia y defecto que promulgan políticas públicas y narrativas locales y globales, con referencia a las personas nombradas con impedimentos. Lo que es peor, estas narrativas retumban en nuestro interior con ínfulas de verdad, eventualmente construyendo subjetividades. Sin embargo, en justicia a lo abarcador del modelo médico clínico y su influencia sobre lo que hoy entendemos en este campo, es importante reconocer, que son muchos los hallazgos bio-médicos, clínicos y de desarrollo de tecnologías de asistir a la vida, que han posibilitado que las personas que nacen con diferencias del cuerpo en el sentido mas amplio puedan acceder a una existencia material en donde reclaman sus derechos, incluyendo el derecho a ser diferente. De esta forma aclaro, que no es la intención, en este trabajo, descartar el modelo médico clínico, sino ubicarlo en su justa perspectiva, sin que sea el fundamento para todo entendido sobre el fenómeno social de las llamadas discapacidades humanas. El modelo médico ayuda a entender qué es lo que hace el cuerpo diferente y cómo se pueden maximizar las vías de comunicación y acceso al entorno físico y social en el cual circulan las personas que no son entendidas con estas diferencias. Sin embargo, no puede ser la única forma de acercarnos a las diferencias humanas porque su solución implica la eliminación de la diferencia o sea la invisibilidad del sujeto con impedimentos y discapacidades del entorno social. Aquí surge un puente muy interesante con otras diferencias humanas entre ellas la de identidad sexual. Las personas gay, lesbianas, bisexuales y transgénero son bienvenidas al ejercicio de la ciudadanía plena siempre y cuando dejen su diferencia fuera, o sea la invisibilizen. Tan pronto se permiten el asumirse tal cual son y se sienten ser, la puerta se cierra dejándoles en los márgenes con los demás que son los otros (Calhoun, 1996).

El tema de los impedimentos humanos se ha abordado desde otras perspectivas dentro de las cuales recientemente se ha elaborado una teoría social de la discapacidad (Oliver, 1996; Shakespeare \& Watson, 1997) en la cual se entiende que no es la persona la que tiene un impedimento sino que es el entorno social quien le impide ejercer sus derechos por no estar preparado para incluirle. Esta teoría advierte las discapacidades con las cuales debe manejarse una persona de cuerpo diferente y los impedimentos 
de carácter social estructural que le son impuesta por una sociedad 'habilista' o sea que apuesta a una forma privilegiada del cuerpo y su funcionamiento al cual se le adscribe el título de norma (Corker \& French, 1999). En otras palabras en la teoría del modelo social, se responsabiliza a la sociedad que no provee un espacio equivalente a las personas con cuerpos que difieren de la norma, de los impedimentos que deben enfrentar estas personas. Queriendo decir que los impedimentos son los obstáculos que enfrentan a nivel socialestructural.

Dentro del entendido amplio de que no es el defecto sino la diferencia lo que distingue a la persona con impedimentos coexisten variadas formas de acercase al entendido de esto que llamamos discapacidades. Entre ellas y muy recientemente está el análisis crítico del discurso (Fairclough, 1995; Corker \& French, 2002). De vital importancia en un análisis crítico del discurso de la discapacidad humana es entender que lo que es, no permanece, el cambio es inminente en casi todo lo que comprende nuestro mundo. Así que lo que es esperado en una cultura como lo capacitado o habilitado en un momento, no lo será en otro tiempo necesariamente, en tanto lo que es valorado cambia. De un solo golpe lo que no es valorado y lo que significa, el sentido de lo deficiente, también cambia.

¿Como cambia la forma en que se construye la identidad de la persona con impedimentos? Con la palabra, en las prácticas discursivas que acompañan la cotidianidad, la formalidad y la virtualidad de la sociedad contemporánea. Judith Butler (2000) en su libro Cuerpos que Importan, explica; cómo los sentidos que tienen las palabras pueden cambiar dramáticamente su performatividad haciendo uso del ejemplo del movimiento Queer, ésta señala que la palabra queer (que se refiere a homosexual pero que significa extraño, raro) y que nace como un insulto dirigido a señalar la deficiencia en la diferencia de orientación sexual. Lo que fue un insulto se convierte en una afirmación de identidad en tanto grupos activistas a favor de los derechos civiles de gays y lesbianas acuñan el término queer para identificarse, cambiando la performatividad de la palabra las opciones de los sujetos nombrados son más amplias. De ahí surge la necesidad de un movimiento de trabajos académicos, investigativos, culturales, político-activistas y sociales de afirmación del derecho a la diferencia, sin que ello implique inferioridad. En el caso de las personas con impedimentos como grupo señalado y marginado por sus diferencias, sería importante ver cual ha sido la evolución de esta performatividad del discurso en torno a los impedimentos humanos. 
Siguiendo la tradición foucaultiana del análisis genealógico, Henri-Jaques Stiker (1998) presenta un recuento de las prácticas discursivas sobre impedimentos humanos desde un contexto europeo y norteamericano; también analiza la evolución del discurso, el cambio en el sentido de las palabras y la eliminación y reforma de muchas palabras en el discurso sobre impedimento humano. La revolución por la equidad social de las personas con impedimentos ha sido una revolución de palabras y significados como ninguna otra. Terribles categorías como; mongoloide, anormal, cojo, bizco, tarado, virado, ya son entendidos como peyorativos y denotan ignorancia y falta de sensibilidad.

Los cambios en el discurso, ya sean por omisión o revolución deben mantener el enfoque en ¿cuál es el efecto que tienen estas prácticas discursivas en cuanto al sujeto que interiorizan todas las personas con relación a lo que son los impedimentos humanos? ¿qué significa ser nombrado discapacitado o impedido? El discurso contemporáneo de la discapacidad la significa como desviación, en tanto que la materialización de la norma del cuerpo no tiene lugar para lo diferente. Samuels (2000) señala que la materialización de la norma es de un cuerpo, definido como el 'cuerpo normal', fuera de lo cual todo es anormal, o menos que normal. En forma similar, Butler (2000) expone los peligros del discurso dominante sobre los cuerpos 'adecuados' y los que no lo son, relegando al reino de lo no válido los cuerpos que no cumplen las 'expectativas' sociales. En un artículo que produce una crítica a los planteamientos de Butler, Samuels (2002) presenta la teoría de ella como aplicable al entendido sobre discapacidad. Sin embargo, Butler no hace mención de esta población en sus escritos. Samuels (2002) expone la aplicabilidad de esta noción de la performatividad de los cuerpos, atado a la construcción social, representado por el lenguaje que se refiere a los cuerpos en tanto a su valor o no valor. La teorización de Butler, según Samuels, es vital en tanto ésta asume una crítica contundente de las construcciones sociales del cuerpo en la cual los cuerpos no válidos son destinados al rechazo, antes o después de nacer, aborto y eutanasia.

Las justificaciones de índole genéticas que se esgrimen en la contemporaneidad para justificar el proyecto del genoma humano, nos devuleven a una suerte de limpieza. De alguna forma esto parece devolvernos a las tendencias Nazis y de imposiciónes hegemónicas eurocentristas. Las teorizaciones de Butler y de Samuels al igual que los intentos mas recientes de autoras como Corker y French (1999) en el campo del análisis discursivo, son rutas necesarias para entender las implicaciones 
de los discursos en el campo de lo médico y científico y sobre todo de la nueva amenaza de extermino vía interferencia genética o no intervención médica, conocida también como eutanasia selectiva.

\section{Origen y breve cronología del Proyecto de la CDPI}

La pieza legislativa que culmina como la Ley de Puerto Rico \#238, Carta de Derechos de las Personas con Impedimentos en Puerto Rico del 31 de agosto del 2004, inicia su trayectoria cómo proyecto de ley Proyecto de la Cámara 4134 del 16 de octubre de 2003. El mismo es de la autoría del Representante a la Cámara Licenciado Víctor García San Inocencio. El proyecto número 4134, referido a la Comisión de Bienestar Social de la Cámara en la misma fecha de radicación y son efectuadas cuatro sesiones de vistas públicas para discutir el mismo en este cuerpo. El 10 de mayo de 2004 pasa por votación unánime. El 13 de mayo de 2004 el P C4134 fue enviado al Senado de Puerto Rico y referido a la Comisión de Bienestar Social de este cuerpo, y finalmente aprobado el 10 de junio del 2004. El 30 de junio es firmado por los presidentes de la Cámara de Representantes y el Senado de Puerto Rico y el 4 de agosto de 2004 es enviado a la Gobernadora en ese momento Hon. Sila María Calderón. El 31 de agosto de 2004 es firmado por la Gobernadora como la Ley de Puerto Rico \#238 con efectividad inmediata. A dos años y medio de su aprobación ${ }^{4}$ y puesta en vigor, el Gobierno de Puerto Rico representado por los representantes de todas las entidades del Estado, los Municipios y Organizaciones No gubernamentales han sometido sus planes estratégicos para su implantación bajo la guía y asistencia técnica de la Procuraduría de las Personas con Impedimentos. Es importante incluir en este análisis que la legislación aprobada no incluye una asignación presupuestaria para implantar las políticas surjan de la misma.

\section{CDPI en el contexto de los derechos humanos}

El gobierno de Puerto Rico representados en éste caso por las ramas Legislativa y Ejecutiva conciben una 'Carta de Derechos para las Personas con Impedimentos', en el 2004, cuando las Naciones Unidas había iniciado una campaña de alerta y atención a los ciudadanos con impedimentos del

\footnotetext{
${ }^{4}$ La Ley de Puerto Rico \#238, Carta de Derechos de las Personas con Impedimentos es aprobada y entra en vigor en Agosto del 2004. En la ley se estipula un periodo de 18 meses para el desarrollo de planes estratégico para la implantación de la política pública que delinea la ley.
} 
planeta en el 1982. La Asamblea General de la Naciones Unidas declaró en 1981 el Año Mundial de los Impedidos y en 1982 aprueba el Programa de Acción Mundial para los Impedidos (Arnade \& Häfner, 2005). ¿A que se debe este lapso en el reconocimiento de los derechos ciudadanos de este sector de la población? Es importante aclarar que aunque se presentaron y aprobaron una variedad de leyes con la intención de beneficiar a las personas con impedimentos, ninguna de ellas hace una declaración de los derechos de las personas con impedimentos como categoría aparte. ¿Por qué una Carta de Derechos para la Personas con Impedimentos? Ello es relevante para entender el contexto particular en el que se da la aprobación de esta Carta de Derechos, para así intentar analizar las construcciones que se producen en torno a estos ciudadanos y ciudadanas que acaban de recibir su título particular de ciudadanos ya entrados en el siglo 21.

En el campo del activismo político se esgrimen los derechos humanos como instrumentos para promover el reconocimiento de parte de las autoridades locales e internacionales de los derechos a la ciudadanía plena de parte de grupos marginados social, política y económicamente.En el caso de las personas con discapacidad se da un sistema de doble acceso de trato legal. Por un lado se ofrecen derechos básicos humanos de participación y autodeterminación a aquellos que cumplen con el requisito del pensamiento racional e independencia, cuya validación en las personas con impedimentos la da el aparato médico-clínico, y por otro lado se ofrecen derechos especiales que conllevan la exclusión política y social (Minow, 1990; 146). Existe primordialmente una Declaración de Derechos Humanos Universales de las Naciones Unidas que data del 1948, que supone proclamar la "igualdad entre todos los miembros de la familia humana" (Organización de las Naciones Unidas, 1948). En ningún lugar de esta declaración encontramos que los derechos que otorgan estos documentos están condicionados a que la persona sea considerada apta para ejercerlos. Se menciona en la Declaración Universal de los Derechos Humanos en el Artículo 7: "Todos son iguales ante la ley y tienen, sin distinción, derecho a igual protección de la ley. Todos tienen derecho a igual protección contra toda discriminación que infrinja esta Declaración y contra toda provocación a tal discriminación" (Naciones Unidas, 1948). Es así también en la Constitución del Estado Libre Asociado de Puerto Rico (1952) establece:

Dignidad e igualdad del ser humano; discrimen, prohibido. La dignidad del ser humano es inviolable. Todos los hombres son iguales ante la Ley. No podrá establecerse 
discrimen alguno por motivo de raza, color, sexo, nacimiento, origen o condición social, ni ideas políticas o religiosas (Artículo II, Carta de Derechos, Sección 1).

La CDPI en su intención de otorgar derechos ciudadanos a las personas con impedimentos los excluye de los Derechos Humanos adquiridos por los demás miembros de su especie. La pregunta que esto obliga es ¿Por qué una Carta de Derechos de las Personas con Impedimentos cuando como miembros de la familia humana según expresa la Declaración Universal de los Derechos Humanos tienen estos derechos desde su nacimiento? Young (1990) señala que la práctica de homogenización obligatoria para acceder a los derechos ciudadanos de parte de los grupos minoritarios, lo que hace es promover el privilegio de unos grupos y la opresión de otros. Esta autora expone la idea de una ciudadanía diferenciada en la que se excluye por tener una identidad socio-cultural diferente. La opresión se puede dar en cinco formas: explotación, marginación, privación de poder, imperialismo cultural y violencias y acosos sistemáticos motivados por el odio o el miedo a los grupos oprimidos (Young, 1990). El modelo médico ha servido como vehículo para el progreso en cuanto a mejorar la calidad de vida de las personas nombradas con impedimentos, a su vez ha sido una fuente de discurso que ha ejercido poder de nombrar a unas personas habilitadas y otras inhabilitadas. La opresión surge de la experiencia de vivir como los y las no ciudadanas/os, dentro de unas practicas discursivas que no son de los y las privilegiadas.

\section{Acceso privilegiado al discurso}

El discurso hegemónico sobre los impedimentos ha estado dominado por el modelo médico-clínico y legitimizado por lo legal. Esto es algo que la CDPI ejemplifica a la perfección. Los derechos otorgados por la CDPI tienen un requisito de elegibilidad, cuya determinación se ha mantenido en manos de las autoridades médicas desde principios del siglo pasado. En el siguiente texto de la CDPI vemos un ejemplo de muchos: "El respeto a sus derechos individuales, limitando el ejercicio de los mismos sólo cuando sea necesario para su salud y seguridad, y como medida terapéutica por un médico debidamente autorizado" (CDPI, Artículo 3, g).

La ciudadanía que promete la CDPI, es una condicionada a la ausencia de las diferencias y al cumplimiento con los requisitos de la cura impuestos por las 
autoridades médico-clínicas. En diversos textos de la CDPI se presentan las condiciones de la ciudadanía de las personas con impedimentos y en cada una de las instancias aparece bajo la discreción de las autoridades médicas. Otros ejemplos de los textos de la CDPI que ilustran la ciudadanía condicionada:

(p) No ser objeto de restricción involuntaria en hospital, hogar sustituto o residencial a menos que exista una orden médica o legal que así lo disponga o que sea necesario por razón de mediar un estado de emergencia para evitar lesiones infligidas a sí mismo o a otros, con sujeción a la legislación o jurisprudencia vigente (CDPI, Artículo 4).

(u) No ser objeto de medicación excesiva con la intención de restringirlo, coartarlo o inmovilizarlo a menos que existan condiciones de salud recurrentes y que atenten contra su seguridad física o de otros, con sujeción a la legislación o jurisprudencia vigente (CDPI, Artículo 4).

Entonces hablamos de una ciudadanía 'especial', que significa asistida y que sin la asistencia no puede ser ejercida. La asistencia y el apoyo que promete la CDPI para que las personas con impedimentos ejerzan sus derechos ciudadanos es prescrita por elementos externos a estas personas. De nuevo las autoridades médico-clínicas definen cuales son las necesidades y cómo pueden ser satisfechas. La CDPI no otorga a las personas con impedimentos una ciudadanía en igualdad de condiciones de las personas que no son nombradas con impedimentos, sino más bien lo que hace es excluirlos/as de la ciudadanía general para otorgar una suerte de condición 'especial' de ciudadanía.

Algunas relaciones de poder y el nivel de racionalización que se requiere para apoyarlas

El presente análisis de la CDPI revela la necesidad como instrumento de ejercicio de poder tanto en la definición de las necesidades como en la satisfacción de las mismas. El poder lo ejerce quien define las necesidades y sus formas de satisfacción. El poder se refiere al de construir el sujeto necesitado. Fraser (1989) argumenta sobre las necesidades como una especie de discurso político importante. El discurso de las necesidades funciona como medio para la elaboración y respuesta a los reclamos políticos. Según 
Fraser (1989) es un idioma a través del cual se desarrolla el conflicto político y se significan y retan las inequidades. Las necesidades aparecen como un campo de lucha en el que grupos con recursos discursivos desiguales compiten por establecer la hegemonía de su interpretación de necesidades como legítimas (Fraser, 1989). Según esta autora, las necesidades en las sociedades capitalistas tardías están altamente politizadas y no son claros los linderos entre lo político, económico y cotidiano. En la CDPI se produce un sujeto, persona con impedimentos que en resumen es definido por sus necesidades. De hecho en el texto, la palabra necesidades es repetida en decenas de ocasiones todas describiendo la diferencia entre personas con impedimentos y otros ciudadanos. Las necesidades de las personas con impedimentos en la CDPI es base sobre la cual se describe la política pública que a su vez se convierte en un discurso que construye a la persona con impedimentos como necesitado, desvalido, incapaz de cumplir con los requisitos de la ciudadanía sin la asistencia del Estado. Por ejemplo el CPDI, en el artículo 4 establece:

(cc) Recibir una educación y adiestramiento, cuando su condición se lo permita que propenda al pleno desarrollo de su personalidad y que se le reconozcan y respeten sus derechos humanos.

(dd) Respetar su autonomía en todo lo relacionado a los asuntos que afecten su vida, progreso, tratamiento, recuperación y rehabilitación, de acuerdo a su grado de funcionamiento general.

Los sujetos y objetos del discurso y como se diferencian: ¿Dónde están las mujeres con impedimentos en la CDPI? La CDPI presenta un sujeto con impedimentos asexual. A pesar de que en su exposición de motivos se hace mención de todos y todas, en lo que sigue del documento no vuelve a aparecer esta diferenciación, tampoco aparece ninguna otra diferenciación que no sea los aptos y no aptos. En este caso se refiere a aquellas personas que pueden acceder al ejercicio de sus derechos ciudadanos por virtud de haber cumplido con ajustar su diferencia a lo esperado en el entorno social cultural.

Las mujeres no aparecen en el sujeto con impedimentos que produce la CDPI, esto es evidente cuando examinamos los derechos que consignan esta carta y la política pública que asigna al Estado. En ningún lugar hace 
mención de asuntos de política pública que enfrentan las mujeres por ser mujeres como por ejemplo son los derechos reproductivos, discrimen por género y violencia doméstica. En el asunto de los derechos reproductivos se hace evidente el vacío en tanto este ha sido un asunto que ha afectado a miles de mujeres con impedimentos. En su situación no se le reconoce derechos reproductivos porque no se le reconoce sexualidad. Ha sido la política de muchos estados esterilizar a mujeres con impedimentos por el prejuicio de una prole defectuosa y la construcción de la mujer con impedimentos como madre inadecuada (Russell, 1998). En el caso de la violencia doméstica se invisibiliza la doble vulnerabilización de la mujer con impedimentos en las relaciones violentas. En primer lugar su realidad como mujer y en segundo lugar su materialidad física que en muchas ocasiones depende de la asistencia de otro para poder lidiar en un entorno social cultural y físico que no acomoda su cuerpo diferente. La invisibilización de esta diferencia de género no permite el que se elaboren políticas sociales adecuadas para la atención de esta parte de la población, tanto para la prevención como para el manejo efectivo de situaciones de violencia y abuso contra mujeres con impedimentos.

El obviar la diferencia de género obstaculiza el desarrollo de políticas sociales que aborden el discrimen por género que sufren las mujeres en general y que se refleja de igual forma entre mujeres con impedimentos. Datos del Censo del 2000 revelan que las mujeres con impedimentos participan en mayor por ciento de actividades de educación superior, obtienen grados académicos en mayor proporción que sus contrapartes masculinos, pero participan en el campo laboral en menor proporción que los hombres (US Census Bureau, 2000). Estos datos son muy similares a los que se reportan para mujeres que no se nombran con impedimentos. Es evidente que mayor atención a las políticas sociales que promueven la participación en la fuerza laboral, podrían redundar en resultados más positivos para las mujeres con impedimentos y sus familias. La invisibilidad de las diferencias del sujeto con impedimentos contribuye a una visión reduccionista de sus realidades y a reforzar políticas incrementalistas que sólo se construyen sobre una visión desvalida de la persona con impedimento que no representa este sujeto en su incomensurable diversidad y riqueza.

¿Cómo preserva el discurso su derecho socialmente construido de pronunciar la 'verdad' en el contexto humano en que aparece: Las Leyes como vehículo de Preservación y transmisión del discurso? 
$\mathrm{Si}$ bien es el modelo médico clínico quien mayormente aporta a la construcción no privilegiada de la persona con impedimento, ha sido necesario tener la legitmidad de otros campos discursivos y de poder para mantener su hegemonía. La construcción de desvalidez se hace evidente en la CDPI en la mención repetida en el documento sobre las 'necesidades' que presenta la persona con impedimentos para el desarrollo de sus capacidades. La CDPI es un documento legal que representa como versiona lo legal en relación a quien se nombra con impedimentos. Cómo ejemplo, vemos en la definición de elegibilidad la categoría persona con impedimento es otorgada por otros que no son la persona con impedimento. Esto impone un reconocimiento de parte de las autoridades médico-clínicas sin el cual las personas con impedimentos no pueden definirse como tal. El reconocimiento de las autoridades con el poder de conferir el estatus de persona con impedimentos, lo mantiene el aparato médico legitimado por lo legal. Las autoridades médicas al conferir el estatus de persona con impedimento lo hacen a través de un diagnóstico que sugiere enfermedad, esto coloca a las personas con impedimento la posición de los que no están bien y que necesitan ayuda. En adición los declara al margen de lo humano ubicándolos en un lugar "especial", que de ninguna forma es una posición de privilegio. En el modelo médico las personas con impedimento pasan de la exhibiciones de fenómenos circenses a los fenómenos de los anales de la medicina, sólo cambia la vitrina (Braddock, citado en Albrecht, et al., 2001).

\section{Conclusión y otras rutas posibles}

Este ejercicio no ha tenido la expectativa de concluir, sino de iniciar un análisis crítico de los instrumentos legales como constructores de subjetividades que excluyen a los diversos y diversas. Es meramente un ejercicio de análisis como tantos otros posibles a la CDPI. El propósito ha sido más bien el de explorar posibles producciones al interior del discurso, su relación con otros textos y discursos en torno a las personas con impedimentos y un intento de entender las formas en que los discursos legales, que luego se convierten en política pública, construyen posibilidades de acceso al ejercicio de la ciudadanía, en poblaciones que han sido llamadas vulnerables y que han sido vulnerabilizadas por medio de palabras. Estas subjetividades, forman parte de los fundamentos que elaboran currículos de profesionales de ayuda como lo son los trabajadores sociales. Trabajadores Sociales, que tienen como misión construir un mundo más justo que incluya la complejidad humana en su inmensa diversidad. Esta reflexión nos obliga 
a otros procesos de mirarnos como profesionales y seres humanos que participamos de alguna forma en las construcciones que luego formulan políticas sociales que promueven la hegemonía de los hábiles sobre los que se consideran deshabilitados.

Otra inquietud ha sido encontrar al sujeto femenino nombrado con impedimentos en el discurso que supone construir su ciudadanía y su existencia humana. Mediante esta ruta intento encontrarla o nombrar su invisibilidad, para ayudar a entender su presente oportunidad de acceso al ejercicio de la ciudadanía, aquella que no será posible sin que pueda ser develada en toda su diversidad. Este es un trabajo que queda inconcluso y que debe ser continuado.

Este ejercicio me devuelve al inicio de la reflexión que me obliga Julia Kristeva (2005), como una invitación que propone que, para iniciar el proyecto de una vida compartida es necesario comprender lo que nos hace diferentes, maravillosamente diversas y diversos.

\section{Referencias}

Arnade, S. y Häfner, S. (2005). Towards Visibility of Women with Disabilities in the UN Convention. Berlin: Disabled Peoples International.

Braddock, David. (2001). En George Albrecht, K. Seelman \& M. Bury, Handbook of Disability Studies. London: Sage.

Calhoun, Chelshire. (1996). Feminism, the family and the politics of the closet: Lesbian and gay Displacement. Oxford: University Press.

Corker, Marianne \& French, Sarah. (2002). Disability Discourse. Philadelphia: Open University Press.

Eroles, Carlos y Ferreres, Carlos. (2002). La Discapacidad: una cuestión de Derechos Humanos. Buenos Aires: Espacio Editorial.

Estado Libre Asociado de Puerto Rico. (1952). Constitución del Estado Libre Asociado de Puerto Rico, Carta de Derechos. Puerto Rico: Autor. 
Fairclough, Norman. (1993). Discourse and Social Change. Cambridge: Polity.

Fairclough, Norman. (1995). Critical Discourse Analisis. London:Longman.

Fraser, Nancy. (1989). Unruly Practices: Power, Discourse and Gender in Contemporary Social Theory.. Minneapolis: University of Minnesota Press.

Foucault, Michael. (1963). The Birth of the Clinic: An Achaeology of Medical Perception. New York: Pantheon Books.

Kristeva, Julia. (2005). At The Limits of Living: To Joseph Grigely. Atenea, XXV,1, 9-15.

Ley de Puerto Rico \#238 del 31 de agosto de 2004, Carta de Derecho de las Personas con Impedimentos de Puerto Rico.

Minow, Margarette. (1990). Making All the Difference: Inclusion, Exclusion and American Law. Ithaca:Cornell University Press.

Mires, Fernando. (2004). El imperialismo norteamericano no existe y otros ensayos. San Juan: Ediciones Vértigo.

Mitchell, Denise \& Snyder, Sam. (1997). The Body and Physical Difference: Discourses of Disability. Ann Arbor: The University of Michigan Press.

Oliver, Max. (1996). Understanding Disability: From Theory to practice. Basingstoke: Macmillan.

Organización de las Naciones Unidas. (1948). Declaración Universal de los Derechos Humanos. New York: Autor.

Powers, Penny. (2001). The Methodology of Discourse Analysis. Boston: Jones and Bartlett.

Rosa Lydia Vélez vs. El Departamento de Educación de Puerto Rico. San Juan: Tribunal de Puerto Rico. 
Russell, Marion. (1998). Beyond Ramps Disability at the end of the Social Contract. Maine: Common Courage Press.

Samuels, Ellen. (2002). Critical Devides: Judith Butlers's Body Theory and the Question of Disability. Journal National Association of Social Work, 14(3): 5-27.

Shakespeare, Tom \& Watson, Tom. (1997). Defending the social model. Disability and Society, 12(2):293-300.

Stiker, Jean Jaques. (1998). A History of Disability. Ann Arbor: University of Michigan Press.

Young, Ivonne Marion. (1990). Justice and the Politics of Difference. New Jersey: Princeton University Press. 
